\title{
Infección y enfermedad por citomegalovirus en niños sometidos a trasplante de órgano sólido. Experiencia en un centro de referencia chileno
}

\author{
M. Paulina Salazar E., Andrea Alba G., Angela Deluchi B., Bessie Hunter M., \\ Jorge Godoy L., Mario Ferrario B., Mario Uribe M. y Erwin Buckel G.
}

\section{Cytomegalovirus infection and disease in pediatric solid organ transplantation. Experience in a Chilean multiorganic transplantation center}

Cytomegalovirus (CMV) infection and disease in transplant (Tx) recipients may severely complicate the patients outcome. Aim: To determine the incidence, clinical characteristics and risk factors for CMV infection and disease in liver and kidney transplant recipients in a tertiary care children's hospital. Method: A clinical and laboratory evaluation was prospectively performed in 44 and 20 children receiving a renal and liver Tx respectively in the Hospital Luis Calvo Mackenna between 2004 and 2006. Results: At the time of the organ Tx 20.3\% (13/64) children were seronegative for CMV. Thirty six per cent (23/64) patients were infected with CMV, of whom $32 \%$ (14/44) received kidney Tx and 9/20 (45\%) received liver Tx. CMV disease occurred in 52\% (12/23) of infected patients. CMV disease was characterized by fever (100\%), anemia (50\%), leucopenia (16.6\%) and specific organ involvement (renal graft $60 \%$ liver graft $57.1 \%$, lung 25\%, intestine $16.6 \%$ ). Variables significantly associated with infection were a CMV seronegative status $(\mathrm{p}=0.035)$ and lower age $5.5+3.7$ years old vs $8.3+4.4$ years old $(\mathrm{p}=0.01)$. Conclusions: Incidence of CMV infection was high in children receiving a solid organ transplant in our institution and near half of infected children developed CMV-associated disease.

Key words: Cytomegalovirus (CMV), infection, solid organ transplantation, pediatrics.

Palabras clave: Citomegalovirus (CMV), infección, trasplante órgano sólido, pediatría.

\section{Introducción}

$\mathrm{E}$ 1 trasplante $(\mathrm{Tx})$ de órgano sólido ha aumentado notablemente su frecuencia durante los últimos años en el Hospital Luis Calvo Mackenna (HLCM), con alrededor de 30 procedimientos anuales. Esta evolución ha traído aparejado un incremento en las patologías propias del paciente inmunosuprimido. Las infecciones, a consecuencia de la inmunosupresión, constituyen una de las principales complicaciones médicas, siendo citomegalovirus (CMV) el agente oportunista más frecuente en estos pacientes ${ }^{1}$.

Citomegalovirus infecta habitualmente a la población sana en las primeras dos décadas de vida, con una seroprevalencia en la vida adulta cercana a 100\%. En el hospedero sometido a Tx la presencia del virus puede ocasionar efectos directos tales como: síndrome febril e invasión de órganos (gastrointestinal, pulmonar, hepático, etc). La replicación viral también produce efectos indirectos tales como: rechazo agudo y crónico del órgano trasplantado, potenciación de la inmunosupresión, desarrollo de infecciones por otros microoganismos oportunistas y a la vez, un incremento en las tasas de mortalidad de estos pacientes $^{1}$.
En los individuos con Tx de órgano sólido sin profilaxis antiviral la infección por CMV generalmente se manifiesta durante los primeros seis meses desde la cirugía del Tx. La fuente de infección es, alternativamente, el traspaso de células infectadas con virus latente desde un donante sero-positivo (infección primaria) o la reactivación de virus presente en el hospedero (infección secundaria) ${ }^{1}$. La replicación viral en ambos casos, es favorecida por la inmunosupresión y por la presencia de mediadores bioquímicos (FNT- $\alpha$, prostaglandinas y catecolaminas) que se liberan en situaciones clínicas propias de los pacientes sometidos a Tx, tales como eventos de rechazo agudo, infecciones, sepsis, falla hepática fulminante y trombosis venosas y arteriales ${ }^{2}$.

Con el conocimiento de la patogénesis de la infección ha sido posible identificar una serie de variables asociadas a la infección y enfermedad por $\mathrm{CMV}$, siendo de máximo riesgo para desarrollar la enfermedad una relación serológica (IgG-CMV) donante (D+)/receptor (R-) $)^{1,3}$. También se incluyen el tipo de órgano trasplantado, la edad del receptor, el grado de inmunosupresión, la eventualidad de rechazo del órgano trasplantado, co-infecciones virales (p. ej: virus herpes 6), bacterianas y fúngicas ${ }^{1}$.

En el concierto mundial la incidencia de infección por
Hospital Luis Calvo Mackenna, Santiago, Chile (MPSE, AAG, ADB, BHM) Clínica Las Condes, Santiago, Chile (JGL, MFB, MUM, EBG)

Recibido: 21 de abril de 2008 Aceptado: 13 de mayo de 2009

Correspondencia a: María Paulina Salazar Encina paulyse@yahoo.com 
CMV muestra gran variabilidad entre centros. Las cifras alcanzan hasta $75 \%$ de infección por CMV durante el primer año post cirugía en pacientes con Tx de órgano sólido y $\sim 44$ a $65 \%$ de enfermedad, en los grupos de mayor riesgo (D+/R-) $)^{3}$. Actualmente, estas cifras han logrado ser disminuidas hasta 9 y $2,5 \%$, respectivamente, en aquellos centros de Tx con mayor experiencia, gracias a la utilización de profilaxis y/o a la modalidad de terapia anticipada (pre-emptive therapy $)^{4,5}$. La gran variabilidad de las incidencias publicadas puede ser explicada no sólo por la experiencia de dichos centros internacionales, sino que también por la presencia de factores que predisponen o aumentan el riesgo de desarrollar la infección.

En Chile, se han comunicado incidencias de $18 \%$ en un estudio multicéntrico de 98 pacientes pediátricos con trasplante renal $^{6}$ y de $29,9 \%$ en 107 niños con trasplante hepático ${ }^{7}$.

\section{Objetivo}

Evaluar, en forma prospectiva, la tasa de infección y enfermedad por CMV en pacientes pediátricos sometidos a Tx de órgano sólido en un centro terciario de derivación nacional, identificando factores asociados a dicha infección.

\section{Pacientes y Métodos}

Se invitó a participar en el estudio a todos los pacientes sometidos a Tx de órgano sólido (hepático o renal) en el HLCM durante los años 2004 a 2006 y que sobrevivieron los primeros 15 días de efectuado el Tx.

Al ingreso se consignó para cada paciente: edad, sexo, patología, tipo de Tx, donante, fecha, y condición inmune específica (IgG) para CMV del receptor.

Se realizó un seguimiento hasta el sexto mes post Tx con las siguientes variables:

- Evaluación clínica y de laboratorio semanal durante el primer mes posterior a la fecha del Tx, y luego mensual con antigenemia para CMV, hemograma, pruebas de función del órgano trasplantado, eventos de rechazo del órgano y su tratamiento, re-Tx, deceso del paciente.

- Caracterización de la infección y de la enfermedad por CMV: Semanas pos-trasplante, valor de antigenemia, criterios definitorios de enfermedad, duración de hospitalización y tratamiento efectuado.

Definiciones (adoptadas de refs. 8-10)

Antigenemia CMV: Detección de pp65 en leucocitos polimorfonucleares (PMNs) de sangre periférica. Se utilizó un anticuerpo monoclonal marcado dirigido contra una proteína de matriz viral (antígeno pp65) Anti Human Cytomagalovirus Immediate Early Antigen (I.E.A.) AD169 (ATCC VR538). ARGENE S.A. France. Se rea- lizó el conteo de células positivas a través de inmunofluorescencia indirecta ${ }^{8}$. Infección: Antigenemia para CMV $(+) \geq 10$ núcleos positivos/400.000 $\mathrm{PMNs}^{9}$. Enfermedad por $C M V$ : incluye el síndrome y compromiso orgánico. Sindrome: antigenemia para CMV (+) y uno o más de los siguientes: temperatura $>38{ }^{\circ} \mathrm{C}$ durante dos días, leucopenia, trombocitopenia, compromiso del estado general, debiendo descartarse otras etiologías. Compromiso orgánico por CMV: Neumonía probable: antigenemia para CMV (+) y signos y síntomas de enfermedad pulmonar, en ausencia de otras etiologías, y/o lavado broncoalveolar (LBA) con cultivo $(+)$, antigenemia $(+)$ o reacción de polimerasa en cadena (RPC) $(+)$. Neumonía definitiva: biopsia pulmonar $(+)$ por inmuno-histoquímica. Enfermedad gastrointestinal: síntomas gastrointestinales, asociados a lesiones de mucosa y demostración de infección por el virus en una biopsia endoscópica. Hepatitis: elevación de bilirrubinemia y o transaminasas hepáticas. Compromiso del $S N C$ : síntomas neurológicos, más la detección de CMV en muestras de LCR, cultivo de LCR o biopsia cerebral. Retinitis: lesión característica confirmada por un oftalmólogo. Compromiso renal: ascenso de creatininemia mayor a $20 \%$ de la basal, sin otra causa que lo explicara. Nefritis: hallazgos histológicos de infección por CMV en biopsia de paciente con disfunción renal. Cistitis: hallazgos histológicos típicos en una biopsia de vejiga obtenida en un paciente con cistitis. La pesquisa del virus en la orina no se consideró diagnóstico de enfermedad.

Aspectos éticos: El protocolo fue aprobado por el Comité de Ética del HLCM y a todos los participantes se les solicitó consentimiento informado (padres) o asentimiento (paciente de 13 años o más).

Análisis estadístico: Los resultados se expresaron en número y porcentaje. Las medias se compararon mediante test de Student no pareado. Comparación de frecuencias test Fisher exact. Programa computacional estadístico Wink 1.

\section{Resultados}

Se incluyeron en el estudio a 64 pacientes, que recibieron 69 trasplantes entre enero 2004 y marzo del 2006 en el HLCM. Dos pacientes fueron excluidos del seguimiento por fallecer antes de 15 días del trasplante (1 hepático y 1 renal). Las características clínicas de los pacientes incluidos se resumen en la Tabla 1.

Las etiologías más frecuentes que originaron el Tx renal fueron las glomerulopatías (n: 17) y la displasia renal (n: 12), y en el caso del Tx hepático correspondieron a atresia de vía biliar (n: 7) y a falla hepática aguda de etiología no precisada (n: 5).

Incidencia de infección por CMV. El seguimiento hasta el sexto mes post Tx con antigenemia para CMV 
permitió obtener una incidencia total de 23/64 infectados (36\%), una frecuencia de $14 / 44(32 \%)$ en el grupo con Tx renal y $9 / 20(45 \%)$ en el grupo con Tx hepático. La incidencia de enfermedad por CMV fue mayor en pacientes con Tx hepático (7/20,35\%) versus aquellos con Tx renal (5/44, 11\%), siendo significativa esta diferencia $(\mathrm{p}=0,032)$. También la proporción de infectados por CMV que cursaron con signos de enfermedad fue mayor en los sometidos a Tx hepático $(7 / 9,78 \%)$ que en los con Tx renal $(5 / 14,36 \%)$.

Las características clínicas generales de los pacientes infectados y de aquellos con criterios de enfermedad se resumen en las Tablas 2 y 3 , respectivamente.

Presentación clínica de la enfermedad. El hallazgo más frecuente fue fiebre (100\%) y luego la disfunción del órgano trasplantado (67\%). Anemia aguda se presentó en $50 \%$. Se detectó compromiso pulmonar en $25 \%$ de los pacientes con Tx hepático. En dos pacientes con Tx hepático se sospechó la existencia de enfermedad gastrointestinal por $\mathrm{CMV}$, uno de ellos con un síndrome diarreico y el otro presentaba una sub-oclusión intestinal; no se realizó biopsia en ellos.

Manejo terapéutico. Se administró ganciclovir endovenoso (10 mg/kg/día) en 22 de los 23 pacientes con criterios de infección. El único que no recibió correspondió a un paciente sometido a Tx renal que tuvo una antigenemia, a los dos días de detectarse la infección, $<10$ núcleos/400.000 PMN.

En cuatro pacientes con Tx hepático se administró inmunoglobulina anti-citomegalovirus por persistencia de signos de laboratorio de infección (antigenemia positiva) pese al tratamiento con ganciclovir.

Un paciente sometido a Tx hepático recibió valganciclovir oral, durante una semana, post alta, a raíz de una enfermedad por CMV de evolución tórpida. La duración del tratamiento tuvo un mínimo de 14 días, suspendiéndose con dos antigenemias para CMV (-)s.

Se utilizó profilaxis con ganciclovir en seis pacientes, dos de los cuales eran IgG-CMV (-); en los cuatro restantes no existió clara justificación. Cinco pacientes presentaron infección por CMV post profilaxis.

Un segundo evento de reactivación de CMV se observó en cuatro pacientes $(17 \%)$. De éstos dos habían recibido Tx hepático y dos fueron sometidos a Tx renal.

Fallecieron durante el seguimiento cuatro (17\%) de los pacientes que habían presentado infección por CMV en los primeros seis meses desde el Tx; de éstos, tres correspondieron a Tx hepáticos y uno a Tx renal. Las causas de muerte fueron las siguientes:

- Paciente 1. Falleció a las 16 semanas del Tx hepático por falla orgánica múltiple, en asociación a neumonía por CMV pesquisada por RPC (+) en LBA.

- Paciente 2. Falleció a las 12 semanas, luego de un tercer Tx hepático, con los diagnósticos de aspergilosis pul- monar, enfermedad por CMV resistente a tratamiento y compromiso pulmonar evidenciado por shell vial $(+)$ en LBA.

- Paciente 3. Falleció a las 24 semanas de Tx hepático por falla orgánica múltiple, en relación a rechazo crónico. Cursó con infección por adenovirus y Aspergillus sp.

- Paciente 4. Falleció a las 15 semanas de Tx renal por falla multiorgánica, en asociación a aspergilosis cerebral y pulmonar. Antecedente de infección por CMV a la semana 12 post-Tx. Desde la cirugía presentó cuatro episodios de reactivación de CMV.

Tabla 1. Características biodemográficas en pacientes pediátricos sometidos a trasplante de órgano sólido. Hospital Luis Calvo Mackenna. 2004-2006 (n: 64)

\begin{tabular}{|c|c|c|c|}
\hline & \multicolumn{2}{|c|}{ Tipo de trasplante } & \multirow[b]{2}{*}{$\begin{array}{l}\text { Total } \\
(64)\end{array}$} \\
\hline & $\begin{array}{l}\text { Renal } \\
\text { (n: 44) }\end{array}$ & $\begin{array}{c}\text { Hepático } \\
\text { (n: 20) }\end{array}$ & \\
\hline Edad promedio, años (rango) & $8,2(2-14)$ & $5,4(1-16)$ & $7,3(1-16)$ \\
\hline Género masculino n (\%) & $(50)$ & $12 \quad(60)$ & $(53)$ \\
\hline Donante cadáver n (\%) & (93) & (95) & (94) \\
\hline $\operatorname{lgG}-\mathrm{CMV}(+)^{*} \quad \mathrm{n}(\%)$ & (75) & (90) & (80) \\
\hline
\end{tabular}

Tabla 2. Características bio-demográficas y evolución de pacientes pediátricos sometidos a trasplante de órgano sólido que adquirieron infección por citomegalovirus. Hospital Luis Calvo Mackenna. 2004-2006

\begin{tabular}{|c|c|c|c|c|}
\hline & \multicolumn{4}{|c|}{ Tipo de trasplante } \\
\hline & & $\begin{array}{l}\text { Renal } \\
\text { (n: 14) }\end{array}$ & $\begin{array}{l}\text { Hepático } \\
\text { (n: 9) }\end{array}$ & Total \\
\hline \multicolumn{2}{|c|}{ Edad (promedio años) } & 5,6 & 3,8 & 5,5 \\
\hline \multicolumn{2}{|l|}{ Sexo (masc/fem) } & $8 / 6$ & $7 / 2$ & $15 / 8$ \\
\hline \multicolumn{2}{|c|}{ Semana diagnóstico ${ }^{1}$} & $7^{a}\left(3-12^{a}\right)$ & $5^{a}\left(1-14^{a}\right)$ & $7^{\mathrm{a}}(1-14)$ \\
\hline \multicolumn{2}{|c|}{ Enfermedad por CMV $\mathrm{n}(\%)^{2}$} & $5(36)$ & 7 (78) & $12(52)$ \\
\hline \multicolumn{2}{|c|}{ Rechazo $^{3} \quad \mathrm{n}(\%)$} & $4(29)$ & $4(44)$ & $8(35)$ \\
\hline \multicolumn{2}{|c|}{ Semana de rechazo ${ }^{4}$} & $1^{a}\left(1-2^{a}\right)$ & $3^{a}\left(1-6^{a}\right)$ & $2^{a}\left(1-6^{a}\right)$ \\
\hline Segundo evento ${ }^{5}$ & $n(\%)$ & $2(14)$ & $2(22)$ & $4(17)$ \\
\hline Fallece ${ }^{6}$ & $n(\%)$ & $1(7)$ & $3(33)$ & $4(17)$ \\
\hline \multicolumn{5}{|c|}{$\begin{array}{l}\text { } 1 \text { Semana promedio y rango al diagnostico de infección por CMV. } \\
2 \text { Pacientes que cumplen criterios de enfermedad. } \\
{ }^{3} \text { Pacientes que presenta rechazo agudo del órgano trasplantado durante el seguimiento. } \\
{ }^{4} \text { Semanas promedio de presentación de rechazo agudo y rango de semanas. } \\
{ }^{5} \text { Pacientes que presenta segundo evento de infección por CMV. } \\
{ }^{6} \text { Pacientes que fallecen durante los } 6 \text { meses de seguimiento. }\end{array}$} \\
\hline
\end{tabular}




\begin{tabular}{|c|c|c|c|c|c|c|c|c|c|c|}
\hline $\mathbf{n}$ & Tx & $\begin{array}{l}\text { Edad } \\
\text { (años) }\end{array}$ & $\begin{array}{l}\text { Semana } \\
\text { post } \mathbf{T x}^{1}\end{array}$ & Fiebre $^{2}$ & Anemia & $\begin{array}{l}\text { Trombo } \\
\text { citopenia }\end{array}$ & Leucopenia $^{4}$ & $\begin{array}{l}\text { Disfunción de } \\
\text { órgano }^{5}\end{array}$ & $\begin{array}{l}\text { Compromiso de } \\
\text { otro sistema }^{6}\end{array}$ & Histología? \\
\hline 1 & $\mathrm{R}$ & 13 & 12 & $\mathrm{Si}$ & $\mathrm{Si}$ & No & $\mathrm{Si}$ & $\mathrm{Si}$ & No & No \\
\hline 2 & $\mathrm{R}$ & 10 & 6 & $\mathrm{Si}$ & $\mathrm{Si}$ & No & No & $\mathrm{Si}$ & No & No \\
\hline 3 & $\mathrm{R}$ & 5 & 7 & $\mathrm{Si}$ & Si & No & No & $\mathrm{Si}$ & No & No \\
\hline 4 & $\mathrm{R}$ & 3 & 6 & $\mathrm{Si}$ & No & No & No & No & No & No \\
\hline 5 & $\mathrm{R}$ & 3 & 12 & $\mathrm{Si}$ & Si & $\mathrm{Si}$ & $\mathrm{Si}$ & No & No & No \\
\hline 6 & $\mathrm{H}$ & 2 & 8 & $\mathrm{Si}$ & $\mathrm{Si}$ & No & No & $\mathrm{Si}$ & Intestinal & No \\
\hline 7 & $\mathrm{H}$ & 5 & 2 & $\mathrm{Si}$ & No & No & No & $\mathrm{Si}$ & No & No \\
\hline 8 & $\mathrm{H}$ & 7 & 6 & Si & No & No & No & $\mathrm{Si}$ & Intestinal & No \\
\hline 9 & $\mathrm{H}$ & 2 & 2 & Si & $\mathrm{Si}$ & No & No & No & No & No \\
\hline 10 & $\mathrm{H}$ & 9 & 14 & $\mathrm{Si}$ & No & No & No & No & Pulmón & LBA- RPC \\
\hline 11 & $\mathrm{H}$ & 1 & 8 & $\mathrm{Si}$ & No & $\mathrm{Si}$ & No & $\mathrm{Si}$ & Pulmón & LBA- S.V. \\
\hline 12 & $\mathrm{H}$ & 3 & 1 & $\mathrm{Si}$ & No & $\mathrm{Si}$ & No & No & Pulmón & No \\
\hline \multicolumn{11}{|c|}{$\begin{array}{l}\text { Tx: tipo de trasplante. R: renal. H: hepático. } \\
{ }^{1} \text { Semanas al diagnóstico de enfermedad por CMV posterior al trasplante. } \\
{ }^{2} \text { Fiebre }>38{ }^{\circ} \mathrm{C} \text {. por lo menos durante } 2 \text { días en un período de } 4 \text { días, se descarta co-infección con otro patógeno. } \\
{ }^{3} \text { Trombocitopenia }<100.000 / \mathrm{mm}^{3} \\
{ }^{4} \text { Leucopenia }<5.000 \text { leucocitos } / \mathrm{mm}^{3} \\
{ }^{5} \text { Disfunción del órgano trasplantado: ascenso creatininemia o aumento de transaminasas hepáticas. } \\
{ }^{6} \text { Compromiso de otro sistema según evolución clínica. } \\
{ }^{7} \text { Diagnóstico histológico. LBA: lavado broncoalveolar. RPC: reacción de polimerasa en cadena para ADN de CMV, SV: shell vial. }\end{array}$} \\
\hline
\end{tabular}

Se analizaron aquellas variables clásicamente asociadas a mayor riesgo de infección por CMV (Tabla 4). Se encontró asociación con el antecedente de serología negativa previa al Tx en el receptor IgG-CMV (-). La edad promedio de los pacientes infectados fue menor que los que no desarrollaron infección 5,5 $\pm 3,7$ años versus

\begin{tabular}{|c|c|c|c|c|}
\hline Factor de riesgo & $\begin{array}{c}\text { Infectados /variable(+) } \\
(\%)\end{array}$ & $\begin{array}{c}\text { Infectados /variable(-) } \\
(\%)\end{array}$ & RR & $\mathbf{p}$ \\
\hline R IgG-CMV(-)' & $8 / 13(62)$ & 15/51 (29) & 2,09 & 0,035 \\
\hline Rechazo $^{2}$ & $8 / 19(42)$ & 15/45 (33) & 1,2 & 0,34 \\
\hline Timoglobulina ${ }^{3}$ & 5/15 (33) & $18 / 49$ (37) & 0,97 & 0,53 \\
\hline Corticoesteroides $^{4}$ & 12/31 (39) & 11/33 (33) & 1,1 & 0,42 \\
\hline \multicolumn{5}{|c|}{$\begin{array}{l}{ }^{1} \text { Serología pre-Tx receptor lgG CMV (-) negativa. } \\
{ }^{2} \text { Evidencia de rechazo del órgano trasplantado durante los } 6 \text { meses de seguimiento. } \\
{ }^{3} \text { Anticuerpos anti-timocito en terapia de rechazo. En } 100 \% \text { de los casos se asoció a profilaxis anti- } \\
\text { CMV con ganciclovir. } \\
{ }^{4} \text { Prednisona, metilpresdnisolona, o prednisolona posterior a los } 7 \text { primeros días de transplante, ya sea } \\
\text { como parte del protocolo de inmunosupresión o terapia de rechazo. }\end{array}$} \\
\hline
\end{tabular}

$8,3 \pm 4,4$ años respectivamente (p: 0,01). Al analizar la edad como factor de riesgo para infección independiente de la condición inmune, se pudo observar que en el grupo IgG-CMV (+) la edad promedio de los que se infectaron era menor que aquellos que no presentaron infección. (T-test independiente, p: 0,034).

\section{Discusión}

En las últimas décadas han existido significativos avances en el conocimiento de la patogénesis de la infección y de la enfermedad por CMV en Tx de órgano sólido, cambiando así las recomendaciones de monitoreo y tratamiento de este agente antes de que se desarrolle la enfermedad y se arriesgue la sobrevida del paciente y del órgano.

En nuestro país hay escasas publicaciones con respecto a la infección por CMV en el paciente sometido a trasplante de un órgano sólido, en particular luego de la incorporación de la antigenemia como método de seguimiento estricto en el período post-trasplante. Los resultados obtenidos en este estudio permiten conocer el comportamiento del virus en una población de pacientes pediátricos sometidos a Tx en el HLCM. 
De acuerdo a las definiciones de infección y enfermedad adaptadas de la $5^{\text {a }}$ Conferencia Internacional de $\mathrm{CMV}^{5}$, en este estudio se reportó una incidencia de infección de $36 \%$ y de enfermedad de $19 \%$ durante los primeros seis meses de seguimiento post Tx hepático y renal. Estos valores son elevados al compararlos con cifras actuales en otros centros de Tx a lo largo del mundo, donde se han logrado reducir la incidencia de enfermedad hasta 9 y $5 \%$ con la utilización de profilaxis ${ }^{4,5,10}$.

Al analizar por órgano trasplantado, es destacable la significativa mayor frecuencia de enfermedad por CMV en pacientes con Tx hepático (35\%) que en aquellos con Tx renal (11\%). Por otra parte, la enfermedad se presentó con mayor gravedad en el Tx hepático, ya que hubo compromiso de otros órganos: pulmón (en tres casos de Tx hepático) e intestino (en dos casos de Tx hepático), si bien no se efectuó confirmación histológica del compromiso de estos órganos en dichos casos. Se evidenció también una mayor tasa de mortalidad en los pacientes sometidos a Tx hepático que presentaron infección por CMV (33\%) durante el seguimiento, versus los sometidos a Tx renal que se infectaron (7\%). Aunque no es posible establecer una relación causa efecto, sí merece destacarse el posible rol del CMV en la evolución del paciente sometido a Tx, principalmente en el Tx hepático. Publicaciones anteriores han atribuido tasas de mortalidad cercanas a $19 \%$ en $\mathrm{Tx}$ hepático con infección por $\mathrm{CMV}^{11}$.

Uno de los objetivos de este estudio fue analizar los factores de riesgo asociados a infección por CMV, tal como condición inmune pre-Tx del donante y receptor ${ }^{5}$. Esta información se obtuvo sólo en los receptores ya que los protocolos utilizados en nuestro país no determinan la serología del donante en forma rutinaria. Las posibilidades de presentar infección y enfermedad aumentan cuando se establece la relación donante positivo/receptor negativo (D+/R-), identificándose en la literatura científica un riesgo hasta $60 \%$ para desarrollar enfermedad ${ }^{11}$. De los 64 pacientes analizados, 20\% eran sero-negativos previo a la cirugía. De estos, $62 \%$ presentaron infección durante el seguimiento. En este estudio se encontró una asociación significativa entre la sero-negatividad del receptor e infección, reproduciéndose lo reportado en series internacionales ${ }^{5}$.

Otro factor analizado fue la edad del paciente sometido a Tx. El promedio de edad fue menor en el grupo que desarrolló infección, hecho esperable ya que a menores edades, mayor es el porcentaje de pacientes con serología negativa para el virus y, por ende, existiría mayor riesgo de infección. Se analizó entonces, el factor edad en aquellos con IgG-CMV (+) pre-Tx, evidenciándose también una edad promedio menor para los infectados. Probablemente en este grupo, la edad representa un factor de riesgo para la reactivación o re-infección viral.

Se han desarrollado innumerables estrategias con el objeto de lograr el delicado equilibrio entre la prevención del rechazo y depresión del sistema inmune ${ }^{12}$. El uso de anticuerpos anti-linfocito e inmunoglobulina anti-timocito también aumenta el riesgo de enfermedad invasora (tres a cuatro veces); ambos suprimen el sistema inmune y, por otro lado, potencian la reactivación del virus latente a través del FNT- $\alpha$, que se libera con su administración. Hoy en día, estos agentes son considerados recursos para profilaxis contra CMV (ver más adelante) ( $^{3,13}$.

Dado que los protocolos de inmunosupresión utilizados en los Tx hepáticos y renales son diferentes, sólo se analizó el uso de corticoesteroides en forma prolongada y el uso de anticuerpos anti-timocito (timoglobulina), que ambos protocolos comparten. El uso de ambos inmunosupresores no constituyó factor de riesgo para infección en esta muestra. Posiblemente este resultado sea atribuible al uso de profilaxis con ganciclovir en $100 \%$ de quienes recibieron timoglobulina.

Debido al gran impacto de la infección por CMV en la evolución post Tx, se han utilizado como estrategia para disminuir el riesgo de infección antivirales e inmunoglobulina anti-CMV en forma profiláctica ${ }^{14}$; no obstante, esto implica prolongar la hospitalización posterior al Tx, utilizar medicamentos no exentos de efectos adversos y toxicidad, aumentar el costo económico, la amenaza de desarrollo de resistencia al antiviral y un aumento, ya documentado, de la incidencia de enfermedad por CMV de presentación tardía. Actualmente la mayoría de los centros internacionales de Tx utilizan la profilaxis en el grupo de mayor riesgo (D+/R-) logrando disminuir el riesgo de enfermedad por CMV hasta $12 \%$ con el uso de ganciclovir oral durante los primeros tres meses posterior al $\mathrm{Tx}^{15}$. Sin embargo, esto ha favorecido el desarrollo de enfermedad por CMV de presentación tardía al suspender la profilaxis y el posible desarrollo de resistencia al antiviral, considerado de elección para el tratamiento de la enfermedad. Valganciclovir, fármaco de uso oral, ha demostrando una eficacia preventiva similar a ganciclovir oral $(9 \%$ de enfermedad por CMV en el grupo de mayor riesgo) y con una excelente bio-disponibilidad; esta profilaxis no evita el riesgo de enfermedad de presentación tardía ${ }^{4}$.

Algunos centros de Tx utilizan la modalidad de terapia anticipada. Ésta se basa en que sobre cierto umbral de viremia la probabilidad de desarrollar enfermedad sintomática aumenta en forma exponencial. Los pacientes son monitorizados, en forma estricta, con mediciones periódicas de la carga viral y al superar el valor de corte establecido, deben recibir terapia antivira ${ }^{10}$. El éxito de esta modalidad requiere de un método diagnóstico de gran sensibilidad, que permita diferenciar los estados de virus latente y virus en replicación. La medición de antigenemia tendría ciertas limitaciones como predictor de enfermedad, tales como: un inadecuado reflejo de la replicación viral, necesidad de contar con suficiente 
número de leucocitos para efectuar la lectura de inmunofluorescencia y ser un examen operador-dependiente. Por otra parte, aún no existe consenso sobre cuál sería el valor de corte para este método. En esta serie clínica se utilizó valor de corte $\geq 10$ núcleos/400.000 PMNs. Hoy en día, en los grupos de más alto riesgo, vale decir D (+)/ R (-), se está utilizando como valor de corte $\geq 2$ núcleos /200.000 PMNs o la confirmación, en el lapso de una semana, de $\geq 1$ núcleo/ 200.000 $\mathrm{PMNs}^{8}$. La RPC en tiempo real (cuantitativa) sería el método ideal, con un valor predictor positivo de $90 \%$ y valor predictor negativo de $95 \%$; sin embargo, su alto costo dificulta disponer de esta técnica en la mayoría de los centros de trasplante ${ }^{8}$. La eficacia de la modalidad de terapia anticipada ha sido demostrada principalmente en grupos de menor riesgo, como aquellos receptores sero-positivo para $\mathrm{CMV}^{4}$.

Al momento de escribirse este manuscrito, aún no existe un protocolo universal de manejo, pero sí se sugiere una estricta monitorización de la carga viral posterior al Tx, y se recomienda el uso de profilaxis con anticuerpo anti-timocito en grupos de riesgo, (D+/R- $)^{4,10}$, y reservar la instauración de terapia anticipada para aquellos receptores seropositivos.

Este estudio presentó algunas limitaciones: el no contar con la serología para CMV del donante, un bajo número de casos reclutados, en especial de Tx hepático, y la falta de diagnóstico histológico en el compromiso órgano-específico, tal como lo establecen las definiciones internacionales ${ }^{4,5}$.

En conclusión, se logró conocer las tasas de infección y enfermedad por CMV en pacientes pediátricos sometidos a Tx de órgano sólido en nuestro país (36 y 19\%, respectivamente) y se confirmaron algunos factores de riesgo conocidos para infección tales como serología negativa en el receptor y menor edad del paciente sometido al trasplante. Los resultados obtenidos sugieren la necesidad de reevaluar los protocolos nacionales de Tx en relación a la prevención y manejo de la infección por CMV.

Proponemos un esquema de monitorización que pueda ser aplicable a las condiciones de nuestro medio nacional:

- Identificar el grupo de riesgo al cual corresponde cada paciente, siendo fundamental para ello el conocimiento de la serología para CMV del donante, cuando el receptor sea IgG-CMV (-).

- En el receptor IgG-CMV (+), adoptar la estrategia de terapia anticipada asociada a la monitorización de antigenemia, idealmente hasta completar 12 meses post-Tx.

- En el receptor IgG-CMV (+), cambio de estrategia a utilizar profilaxis con ganciclovir durante la inducción con anticuerpos anti-timocito o durante el tratamiento de rechazo del órgano.

- En el receptor IgG-CMV (-) con donante IgG-CMV $(+)$ recomendamos la profilaxis con valganciclovir oral hasta completar tres meses post-Tx y luego continuar una monitorización con antigenemia mensual hasta completar 12 meses post-Tx.

- En el receptor IgG-CMV (-) con donante IgG-CMV (-) no se justifica realizar profilaxis ni terapia anticipada.

Agradecimientos: A Oscar Araneda Valenzuela. Al personal de atención en policlínicas de Nefrología y Gastroenterología del HLCM.

\section{Resumen}

La infección y enfermedad por citomegalovirus (CMV) en pacientes sometidos a trasplantes (Tx) es una complicación que condiciona la evolución del injerto y la sobrevida del paciente. Objetivos: Determinar la incidencia de infección y enfermedad por CMV durante los primeros seis meses de efectuados Tx hepático y renal. Caracterizar la enfermedad, e identificar factores de riesgo asociados a infección. Metodología: Análisis prospectivo en 64 pacientes pediátricos sometidos a Tx renal (n: 44) o hepático (n: 20) en el Hospital Luis Calvo Mackenna entre 2004 y 2006. Resultados: Al trasplante, 23,1\% (13/64) eran receptores IgG CMV (-). Cumplieron criterio de infección $36 \%(23 / 64)$ de los pacientes, con Tx renal $32 \%$ (14/44) y con Tx hepático 45\% (9/20). Desarrolló enfermedad el $52 \%(12 / 23)$ la que se caracterizó por fiebre $(100 \%)$, anemia $(50 \%)$, leucopenia $(16,6 \%)$, disfunción del órgano trasplantado $60 \%$ en Tx renal, hepático 57,1\%, compromiso pulmonar en $25 \%$ e intestinal en $16,6 \%$ del total de pacientes. Variables asociadas a infección fueron: ser receptor IgG CMV (-)pre-Tx $(\mathrm{p}=0,035)$ y una menor edad del paciente $5,5+3,7$ vs $8,3+4,4(p=0,01)$. Conclusiones: Hay una elevada tasa de infección por CMV en la población de pacientes con Tx renal y hepática en nuestro medio, la mitad de ellos desarrolló enfermedad amenazando la función del injerto. 


\section{Referencias}

1.- Simon D M, Levin S. Infectious complications of solid organ transplantations. Infect Dis Clin North Am 2001; 15: 521-49.

2.- Kutza A S, Muhl E, Hackestein H. High incidence of active cytomegalovirus infection among septic patients. Clin Infect Dis 1998; 26: 1076-82.

3.- Klara M. Optimal approach to prevent CMV disease. Pediatr Transplant 2008; 12: 385-8.

4.- Sun H Y, Wagener M M, Singh N. Prevention of post-transplant cytomegalovirus disease and related outcomes with valganciclovir: a systematic review. Am J Transplant 2008; 10: 2111-8.

5.- Preiksaitis J K, Brennan D C, Fishman J, Allen U. Canadian Society of Transplantation Consensus Workshop on Cytomegalovirus Management in Solid Organ Transplantation Final Report. Am J Transplant 2005; 5: 218-27.
6.- Cano F, Rosati P, Pinto V, Quiero X, Lagos E, Delucchi A, et al. Trasplante renal en pediatría, una década de experiencia multicéntrica. Rev Chil Pediatr 2001; 72: 505-15.

7.- Uribe M, Buckel E, Ferrario M, Godoy J, González G, Hunter B, Ceresa S, et al. Pediatric liver transplantation: ten years of experience in a multicentric program in Chile. Transplant Proc 2005; 37: 3375-7.

8.- Baldanti F, Lilleri D, Gerna G. Monitoring human cytomegalovirus infection in transplant recipients. J Clin Virol 2008; 41: 237-41.

9.- Ljungman P, Griffiths P, Paya C. Definitions of cytomegalovirus infection and disease in transplant recipients. Clin Infect Dis 2002; 34: 1094 -7.

10.- Humar A, Michaels M; AST ID Working Group on Infectious Disease Monitoring. American Society of Transplantation recommendations for screening, monitoring and reporting of infectious complications in immunosuppression trials in recipients of organ transplantation. Am J Transplant 2006; 6: 262-74.

11.- Campbell A L, Herold B C. Strategies for the prevention of cytomegalovirus infection and disease in pediatric liver transplantation recipients. Pediatr Transplant 2004; 8: 619-27.

12.- Pereyra F, Rubin R. Prevention and treatment of cytomegalovirus infection in solid organ transplant recipients. Curr Opin Infect Dis 2004; 17: 357-61.

13.- Tanaka K. Immunosuppressive agents and cytomegalovirus infection. Arch Immunol Ther Exp 2003; 51: 179-84.

14.- Opelz G, Dohler B, Ruhenstroth A. Cytomegalovirus prophylaxis and graft outcome in solid organ transplantation: A collaborative transplant study report. Am J Transplant 2004; 4: 928-36.

15.- Razonable R. Cytomegalovirus infection after liver transplantation: Current concepts and challenges. World J Gastroenterol 2008; 31: 4849-60. 